\title{
Quantum Mechanics of Gravitational Waves
}

\author{
Maulik Parikh, ${ }^{1,2}$ Frank Wilczek, ${ }^{1,3,4,5}$ and George Zahariade $\oplus^{1,2}$ \\ ${ }^{1}$ Physics Department, Arizona State University, Tempe, Arizona 85287, USA \\ ${ }^{2}$ Beyond Center for Fundamental Concepts in Science, Arizona State University, Tempe, Arizona 85287, USA \\ ${ }^{3}$ Department of Physics, Stockholm University, Stockholm SE-106 91, Sweden \\ ${ }^{4}$ Center for Theoretical Physics, Massachusetts Institute of Technology, Cambridge, Massachusetts 02139, USA \\ ${ }^{5}$ Wilczek Quantum Center and T. D. Lee Institute, Shanghai 200240, China
}

(Received 28 October 2020; accepted 7 June 2021; published 19 August 2021)

\begin{abstract}
For the purpose of analyzing observed phenomena, it has been convenient, and thus far sufficient, to regard gravity as subject to the deterministic principles of classical physics, with the gravitational field obeying Newton's law or Einstein's equations. Here we treat the gravitational field as a quantum field and determine the implications of such treatment for experimental observables. We find that falling bodies in gravity are subject to random fluctuations ("noise") whose characteristics depend on the quantum state of the gravitational field. We derive a stochastic equation for the separation of two falling particles. Detection of this fundamental noise, which may be measurable at gravitational wave detectors, would vindicate the quantization of gravity, and reveal important properties of its sources.
\end{abstract}

DOI: 10.1103/PhysRevLett.127.081602

Introduction.-The behavior of objects falling freely under the influence of gravity is commonly described by Einstein's general theory of relativity, with the curvature field treated classically. Individual test particles follow the geodesic equation while the separations of pairs of test particles obey the geodesic deviation equation. These are deterministic equations, befitting the classical theory from which they are derived. But the fundamental laws of physics are quantum mechanical and, in the context of gravity, we expect the spacetime metric to be a quantum field. To take this into account, a different framework is required.

Here we present a formalism for calculating the effect on falling bodies due to the quantization of the gravitational field. We find that the dynamics of the separation of a pair of falling particles is no longer deterministic, but probabilistic, being acted on by a novel stochastic force. Specifically, we find that the separation of the two particles now obeys a Langevin-like stochastic equation containing a random fluctuation term, or noise [1] (as is further discussed in the Supplemental Material [2] and more fully in Ref. [3]). This provides the quantum generalization of the classical geodesic deviation equation. Our result applies also to a single object falling in the gravitational field of a heavier, fixed mass. Thus, an apple falling in Earth's gravity, say, would not fall straight down but would be

Published by the American Physical Society under the terms of the Creative Commons Attribution 4.0 International license. Further distribution of this work must maintain attribution to the author(s) and the published article's title, journal citation, and DOI. Funded by SCOAP ${ }^{3}$. subject to minute quantum jitters, which can be regarded heuristically as arising due to the bombardment of the apple by gravitons.

This effect is potentially measurable at gravitational wave detectors. We can model the mirrors of an arm of a gravitational wave interferometer [4,5] as two freely falling particles, and couple them to a quantized weak gravitational field. Then, using our formalism (which is based on the Feynman-Vernon influence functional), the effect on the separation of the mirrors can be calculated; the result is that the mirror separation is subject to quantumgravitational noise. Moreover, the unusual power spectrum of this noise can allow it to be distinguished from many other sources of noise that gravitational wave interferometers are susceptible to [6]. The statistical properties of the noise depend on the quantum state of the gravitational field, and we have calculated it explicitly for several classes of states. We estimate that the noise is unmeasurably small for coherent states, which are minimum-uncertainty quantum states that most closely resemble classical gravitational waves. However, there are theoretically predicted, though as yet unobserved phenomena, involving evaporation of black holes and exotic phases in the early Universe, wherein quantum aspects of gravitational radiation play a central role. For the corresponding quantum states, we find that the noise can be significantly enhanced. In particular, in squeezed states the noise can be enhanced exponentially in the squeezing parameter. Detection of this fundamental noise would provide experimental evidence for the quantization of gravity. Finally, we also discuss the connection between features of the radiation sources and the quantum nature of the radiation field. 
Analysis.-We are interested in how a pair of free-falling particles responds to a quantized gravitational field (compare Refs. [7-12]). We refer to the pair as a detector since the two mirrors at the ends of the arm of a gravitational wave interferometer can be idealized as two free-falling massive particles in a weak gravitational field. (This description would hold more literally for a space-based interferometer.) Suppose that the initial state of the gravitational field is $|\Psi\rangle$. We have in mind that this initial state was created by distant astrophysical sources and therefore can be regarded as initially unentangled with the detector state. As the field interacts with the detector, its quantum state changes because the detector generically both absorbs and emits gravitons through spontaneous and stimulated emission; the final field state $|f\rangle$ is a priori unknown. We would like to know the transition probability for the detector to go from state $|A\rangle$ to state $|B\rangle$ in time $T$. Since we do not measure the final state of the gravitational field, we must sum over $|f\rangle$. Thus, we wish to calculate

$$
P_{\Psi}(A \rightarrow B)=\sum_{|f\rangle}|\langle f, B|\hat{U}(T)| \Psi, A\rangle|^{2},
$$

where $|a, b\rangle \equiv|a\rangle \otimes|b\rangle$, and $\hat{U}$ is the unitary timeevolution operator for the combined gravitational field + detector system.

To go further, we need a more detailed description of the observed degrees of freedom; it is significant to focus on observables because the natural variables include unphysical gauge dependence. Let the geodesic separation of the two particles be $\xi(t)$. The dynamics of the combined system of gravity and the two particles is described by the Einstein-Hilbert action minimally coupled to the actions of the two nonrelativistic particles. The weakness of the gravitational field in the radiation zones allows us to expand the particle-field interaction, which is our main interest, in the metric perturbation $h_{i j}$. We will also keep up to quadratic terms in the purely gravitational action. This truncation neglects gravitational self-interaction. It corresponds to summation of a large class of Feynman graphs, as indicated in Fig. 1. Then,

$$
\begin{aligned}
S= & -\frac{c^{4}}{64 \pi G} \int d^{4} x \partial_{\mu} h_{i j} \partial^{\mu} h^{i j} \\
& +\int d t \frac{1}{2} m_{0}\left(\delta_{i j} \dot{\xi}^{i} \dot{\xi}^{j}-\dot{h}_{i j} \dot{\xi}^{i} \xi^{j}\right) .
\end{aligned}
$$

In this expression, we are left with only a single degree of freedom for the two particles: their gauge-invariant separation. Thus, our results apply also to the case of a single particle subject to the gravity of a heavier, fixed mass. We can now evaluate the amplitudes in Eq. (1) in the path integral formulation derived from the action in Eq. (2), where $|A\rangle$ and $|B\rangle$ are the initial and final states of $\xi$; see Fig. 1. Thus, we have

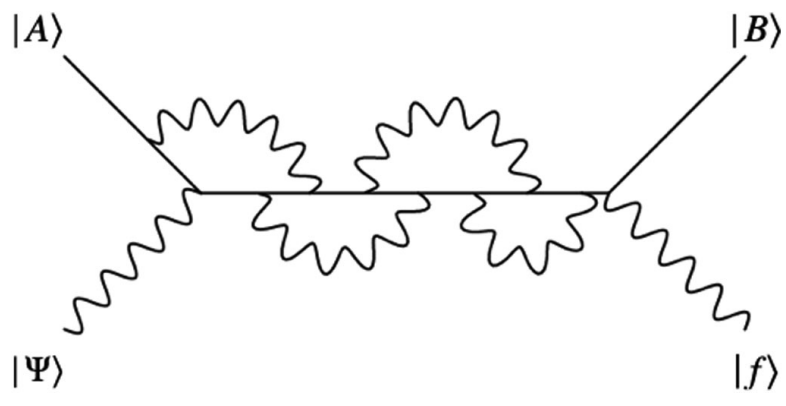

FIG. 1. A generic Feynman diagram representing an elementary process involved in the transition probability in Eq. (1). Solid lines represent the detector while wiggly ones represent gravitons. Notice that, given Eq. (2), the only vertices allowed are graviton-detector-detector vertices which eliminates the possibility of pure graviton loops. Moreover, since the detector is ultimately expected to behave classically, we also disregard pure detector loops.

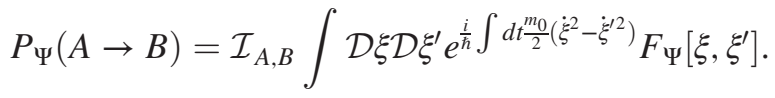

This expression can be understood as follows. The double path integral reflects the fact that this is a probability, rather than an amplitude. The factor $\mathcal{I}_{A, B}$ contains integrals over the initial and final wave functions of $\xi$, and will play no further role. In the exponent, we recognize the nonrelativistic action for a free particle. Equation (3) gives us an effective theory for the particle separation $\xi$, in which the effects of its coupling to the quantized gravitational field have been taken into account. Crucially, all the effects of the quantized gravitational field are formally captured by the functional $F_{\Psi}\left[\xi, \xi^{\prime}\right]$ known as the Feynman-Vernon influence functional [13-15].

To evaluate the influence functional, we write the gravitational field state $|\Psi\rangle$ as a tensor product of singlemode states: $|\Psi\rangle=\bigotimes_{\vec{k}}\left|\psi_{\vec{k}}\right\rangle$. For a weak gravitational field, linearity allows us to treat the problem mode by mode and then sum over modes. Then, $F_{\Psi}\left[\xi, \xi^{\prime}\right]=\prod_{\vec{k}} F_{\psi_{\vec{k}}}\left[\xi, \xi^{\prime}\right]$, where $F_{\psi_{\vec{k}}}\left[\xi, \xi^{\prime}\right]$ is the influence functional for a single mode of the gravitational field. To compute this, we decompose the metric perturbation in Fourier modes. Let the mode of wave number $\vec{k}$ have angular frequency $\omega_{\vec{k}}$ and amplitude $q_{\vec{k}}(t)$. If we now, for simplicity, assume that the perturbation propagates orthogonally to the line joining the particles, and if we further restrict to a single polarization, then we find that the gravitational part of the action in Eq. (2) for a single mode of the gravitational field is

$$
S_{\xi}^{\vec{k}}=\int d t\left(\frac{1}{2} m\left(\dot{q}_{\vec{k}}^{2}-\omega_{\vec{k}}^{2} q_{\vec{k}}^{2}\right)-g \dot{q}_{\vec{k}} \dot{\xi} \xi\right) .
$$

Here, $g=m_{0} / 2 \sqrt{\hbar G / c^{3}}$ is a coupling constant, while $m$ is a mass introduced for dimensional reasons; $m$ depends on 
an infrared cutoff scale and will drop out of all physical expressions after integration over modes.

The model can be made more realistic by including the other polarization, as well as by properly accounting for various trigonometric factors that would arise for modes that are incident from different directions; here we neglect those complicating refinements to focus on the core problem.

Notice that the single-mode action in Eq. (4) describes a simple harmonic oscillator of angular frequency $\omega_{\vec{k}}$ coupled to an external source $\xi$, via a cubic, derivative interaction. We can readily quantize this simple action to evaluate the influence functional; the calculation can be performed, without further invoking perturbation theory, because the Lagrangian is quadratic in $q$. The associated quantum Hamiltonian reads $\hat{H}_{\xi}^{\vec{k}}=\left(\hat{p}_{\vec{k}}+g \xi \dot{\xi}\right)^{2} / 2 m+(1 / 2) m \omega_{\vec{k}}^{2} \hat{q}_{\vec{k}}^{2}$. Correspondingly, the expression for the single-mode influence functional is $F_{\psi_{\vec{k}}}\left[\xi, \xi^{\prime}\right]=\left\langle\psi_{\vec{k}}\left|\hat{U}_{\xi^{\prime}}^{\vec{k} \dagger}(T) \hat{U}_{\xi}^{\vec{k}}(T)\right| \psi_{\vec{k}}\right\rangle$, where $\hat{U}_{\xi}^{\vec{k}}$ is the unitary time-evolution operator obtained from $\hat{H}_{\xi}^{\vec{k}}$. Repeated application of the Baker-CampbellHausdorff formula then yields

$$
F_{\psi_{\vec{k}}}\left[\xi, \xi^{\prime}\right]=F_{0_{\vec{k}}}\left[\xi, \xi^{\prime}\right]\left\langle\psi_{\vec{k}}\left|e^{-W^{*} \hat{a}^{\dagger}} e^{W \hat{a}}\right| \psi_{\vec{k}}\right\rangle,
$$

where $W$ and $F_{0_{\bar{k}}}\left[\xi, \xi^{\prime}\right]$ are readily calculated functions of $\xi$ and $\xi^{\prime}$. Equation (5) is useful computationally because the exponents are expressed in terms of ladder operators $\hat{a}$ and $\hat{a}^{\dagger}$, whose operation on $\left|\psi_{\vec{k}}\right\rangle$ can be calculated for many classes of states. In particular, Eq. (5) can be evaluated in closed form for vacuum, coherent, thermal, and squeezed states.

Having obtained the influence functional for a single mode of the gravitational field in an initial state $\left|\psi_{\vec{k}}\right\rangle$, we are now ready to sum over modes to find the total influence functional of the gravitational field from the product of single-mode influence functionals. The result will depend on the quantum state of the gravitational field. As a basic example, suppose the gravitational field is in its vacuum state. Performing the mode sum, we find in particular that

$$
\begin{aligned}
\ln \left|F_{\mathrm{vac}}\right|= & -\frac{m_{0}^{2}}{32 \hbar^{2}} \int_{0}^{T} \int_{0}^{T} d t d t^{\prime} A\left(t-t^{\prime}\right) \\
& \times\left[X(t)-X^{\prime}(t)\right]\left[X\left(t^{\prime}\right)-X^{\prime}\left(t^{\prime}\right)\right],
\end{aligned}
$$

where $X=d^{2}\left(\xi^{2}\right) / d t^{2}, X^{\prime}=d^{2}\left(\xi^{\prime 2}\right) / d t^{2}$, and $A\left(t-t^{\prime}\right)$ is a known integral. Feynman and Vernon realized that whenever $\ln |F|$ is quadratic in $X-X^{\prime},|F|$ can be rewritten in a very suggestive manner, as a statistical average over an auxiliary function $N(t)$ :

$$
\left|F_{\mathrm{vac}}\right|=\left\langle\exp \left(\frac{i}{\hbar} \int_{0}^{T} d t \frac{m_{0}}{4} N(t)\left[X(t)-X^{\prime}(t)\right]\right)\right\rangle_{N} .
$$

Here the function $N(t)$ obeys a Gaussian probability distribution with a stationary autocorrelation function $A\left(t-t^{\prime}\right)$. Thus, the effect of coupling to the quantumgravitational vacuum, which is fully encoded in $F_{\text {vac }}$, is to introduce stationary stochastic noise in the detector. As we will see, this creates fluctuations in the length of the arm. All the statistical properties of the fluctuations, such as the standard deviation, can be obtained from the autocorrelation function, $A\left(t-t^{\prime}\right)$. In contrast, we find that the phase of $F_{\text {vac }}$ has a different structure, which is responsible for dissipative effects. For a coherent state corresponding to a classical gravitational wave $h(t)$, the phase of the influence functional also contains a term $i / \hbar \int_{0}^{T} d t(1 / 4) m_{0} h(t)\left[X(t)-X^{\prime}(t)\right]$.

Quantum geodesic deviation.-We have so far focused on the Feynman-Vernon influence functional for a detector coupled to a gravitational field, treating both the detector and the field quantum mechanically. Since realistic detectors are well approximated as classical, it is appropriate to exploit that simplification. In our expression for the transition probability, Eq. (3), we identify the classical paths, which dominate the integral, as those which render the phases stationary. This leads to an effective stochastic equation of motion for the separation of the masses or, equivalently, for the arm length of a gravitational wave detector. In the presence of a classical gravitational wave $h(t)$, we find

$$
\ddot{\xi}(t)-\frac{1}{2}\left[\ddot{h}(t)+\ddot{N}(t)-\frac{m_{0} G}{c^{5}} \frac{d^{5}}{d t^{5}} \xi^{2}(t)\right] \xi(t)=0 .
$$

Thus, the relative acceleration of the two masses $\ddot{\xi}$ depends on three terms. Each term in this equation, which extends the geodesic deviation equation of general relativity to the case where the spacetime metric is treated as a quantum field, has intuitive meaning. The first represents the usual tidal acceleration due to the passing of a classical gravitational wave $h$; this is the effect that has been famously measured at LIGO. The last term is the gravitational counterpart of the dissipative Abraham-Lorentz term in electromagnetism; it is the gravitational radiation reaction [16-19]. Although it is likely to be of little experimental consequence, it is nevertheless notable that such a term arises from a well-behaved quantum theory. (As will be reported elsewhere, this approach to radiation reaction avoids the notorious pathologies that arise from too literal interpretation of the Abraham-Lorentz equation [20]). Most importantly, our equation contains a noise term $\ddot{N}$. Thus, our equation, while classical, is stochastic rather than deterministic $[14,15]$. It is reminiscent, mathematically, of the Langevin equation used to describe Brownian motion.

We have calculated the statistical properties of this noise, specifically the power spectrum $S(\omega)$, in several cases; a precise calculation of the quantum-gravitational power 


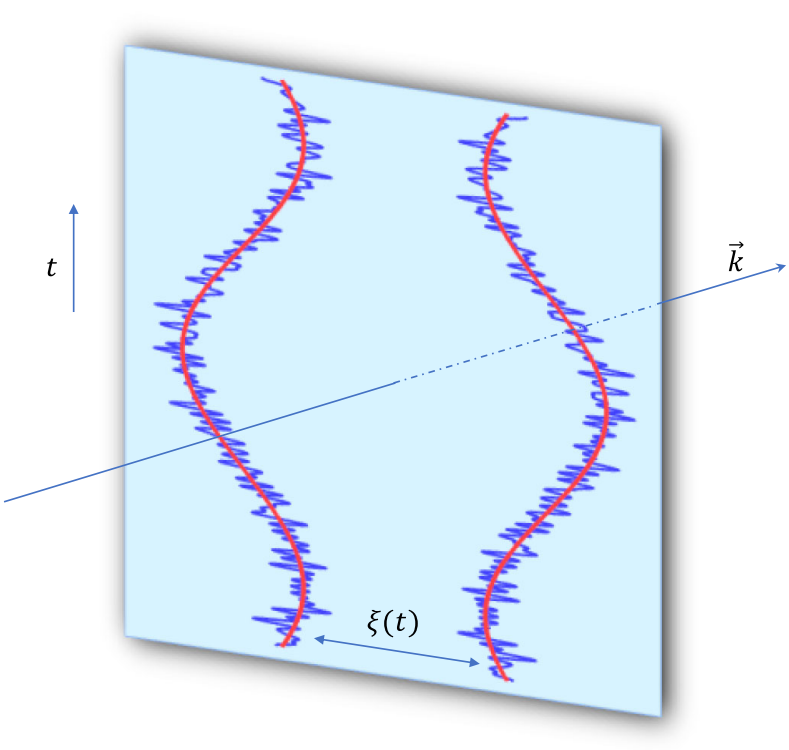

FIG. 2. Schematic spacetime diagram of the motion of the end points of the detector in the presence of one polarization of a quantized gravitational wave propagating along $\vec{k}$. On average, each particle traces an inertial trajectory following a geodesic of the classical spacetime. The physical separation $\xi(t)$ of the two particles obeys, on average, the geodesic deviation equation. The effect of quantization of the gravitational field is to introduce a random fluctuation on top of this classical motion.

spectrum is essential to distinguish the noise from other less fundamental sources of noise at gravitational wave detectors $[21,22] . S$ is the Fourier transform of the autocorrelation function $A\left(t-t^{\prime}\right)$. For the vacuum and for coherent states, we find that, for low frequencies, the power spectrum behaves like $S=4 G \hbar \omega / c^{5}$. For thermal states at temperature $T$, we find $S=4 G \hbar \omega / c^{5} \operatorname{coth}\left(\hbar \omega / 2 k_{B} T\right)$. Strikingly, if the gravitational field is in a squeezed state with squeezing parameter $r$, we find that the noise also contains a nonstationary piece; focusing on the stationary part, we find $S=\sqrt{\cosh 2 r} 4 G \hbar \omega / c^{5}$, which means that the quantum-induced fluctuations of the detector arm length can be exponentially enhanced.

Discussion.-We have shown that a pair of freely falling particles in a quantized gravitational field obeys the stochastic equation (8), rather than the classical geodesic deviation equation; see Fig. 2. Let us estimate the size of the fluctuations, neglecting the radiation reaction term. Then, $\xi(t) \approx[1+(h+N) / 2] \xi_{0}$, where $\xi_{0}$ is the equilibrium length of the arm; for LIGO, $\xi_{0} \approx 4 \mathrm{~km}$, while for LISA, $\xi_{0} \approx 10^{6} \mathrm{~km}$. As the formula makes clear, the fluctuations of $N$ induce fluctuations in $\xi$. We find that $\langle\xi\rangle=(1+h(t) / 2) \xi_{0}$, with a variance $\sigma^{2}=\xi_{0}^{2}\left\langle N^{2}\right\rangle / 4=$ $\xi_{0}^{2} A(0) / 4$. Although $A(0)=1 / \pi \int_{0}^{\infty} d \omega S(\omega)$ is formally divergent, the size of the fluctuations is nevertheless finite because limits on detector sensitivity impose a cutoff $\omega_{\max }$ on the frequency integral; for LIGO, $\omega_{\max } \sim 1 \mathrm{kHz}$, while for LISA, $\omega_{\max } \sim 0.1 \mathrm{~Hz}$. With these numerical values, the amplitude of the fluctuations in the vacuum state $\sigma \sim$ $l_{P} \xi_{0} \omega_{\max } / c$ is roughly a Planck length $l_{P}$, and therefore, completely unobservable; essentially, the same amplitude is obtained also when the gravitational field is in a coherent state corresponding to a classical gravitational wave propagating in the vacuum [9].

However, the amplitude of the fluctuations can be enhanced for noncoherent states of the gravitational field. As representative examples, we may consider states formed by the action of displacement operators representing the classical field $h$ acting not upon the vacuum, but upon thermal or squeezed states. For thermal states at temperature $T$, we have found an enhancement by a factor $\sqrt{2 k_{B} T / \hbar \omega_{\max }}$. Such thermal states could arise, for example, through the isotropic cosmic gravitational wave background $(T \sim 1 K)$ or as a result of the Hawking evaporation of black holes [23]. In the latter case, the temperature can be very high, but there is an additional suppression due to the localization of the source. Most promisingly, there can be significant noise for squeezed states. As noted above, we expect the amplitude of fluctuations to be enhanced exponentially in the squeezing parameter $\sigma \sim e^{r / 2} l_{P} \xi_{0} \omega_{\max } / c$. Physically, gravitational squeezed states can have different possible origins. Squeezed vacua can arise in inflationary cosmology [24-26]. But in addition, squeezed states are naturally produced by classical sources through the nonlinearities of gravity, without assuming any exotic physics.

Indeed, it is enlightening to compare the quantum nature of electromagnetic and gravitational radiation fields for known and contemplated sources; the quantum noise of photons on the dynamics of charged particles is analogous and of course the subject of quantum optics. It is almost always appropriate to treat the coupling of the electromagnetic fields to its sources as linear. When one has linear coupling of a radiation field to dynamical degrees of freedom which are described, to a good approximation, as deterministic and only weakly perturbed by the radiation, then the radiation field will be well described by a coherent quantum state [27]. This is the case for most radio and microwave sources and for lasers. When the sources themselves are stochastic, one obtains a stochastic mixture of coherent states. This is the case for the most common (quasithermal) higher frequency sources. With special techniques, e.g., using nonlinear crystals, one can construct sources whose coupling to the electromagnetic field is quadratic, leading to squeezed states. The default "classical" treatment of gravitational radiation, which corresponds to coherent states, is appropriate when the sources are governed by approximately deterministic dynamics involving weak linear coupling to the gravitational field. Here, in effect, one expands the quantum action around a classical solution of the Einstein-Hilbert equations (including flat spacetime in the radiation zone). This is 
often an appropriate default, e.g., in describing slow orbital decay of large bodies. It does not apply straightforwardly to Hawking radiation, which is a quasithermal quantum process, or to its cosmological analogs or, more speculatively, to phase transitions in the early Universe. During the late stages of black hole mergers, the approximation of treating gravitational radiation as a weak linear perturbation is not appropriate, despite the deterministic nature of the dynamics. Here one can expect to encounter effects we might call molding of the quantum radiation state, which go beyond (quadratic) squeezing.

We thank Paul Davies, Bei-Lok Hu, Phil Mauskopf, Siddharth Morampudi, Igor Pikovski, and Tanmay Vachaspati for conversations. During the course of this work, M.P. and G.Z. were supported in part by John Templeton Foundation Grant No. 60253. G.Z. also acknowledges support from the Foundational Questions Institute and Moogsoft. F. W. is supported in part by the U.S. Department of Energy under Grant No. DE-SC0012567, by the European Research Council under Grant No. 742104, and by the Swedish Research Council under Contract No. 335-2014-7424.

[1] M. Parikh, F. Wilczek, and G. Zahariade, The noise of gravitons, Int. J. Mod. Phys. D 29, 2042001 (2020).

[2] See Supplemental Material at http://link.aps.org/ supplemental/10.1103/PhysRevLett.127.081602 for more detailed derivations of the equations in this letter.

[3] M. Parikh, F. Wilczek, and G. Zahariade, companion paper, Signatures of the quantization of gravity at gravitational wave detectors, Phys. Rev. D 104, 046021 (2021).

[4] B. P. Abbott et al. (LIGO Scientific and Virgo Collaborations), Observation of Gravitational Waves from a Binary Black Hole Merger, Phys. Rev. Lett. 116, 061102 (2016).

[5] P. Amaro-Seoane et al. (LISA Collaboration), Laser interferometer space antenna, arXiv:1702.00786.

[6] B. P. Abbott et al. (LIGO Scientific and Virgo Collaborations), GW150914: The Advanced LIGO Detectors in the Era of First Discoveries, Phys. Rev. Lett. 116, 131103 (2016).

[7] G. Amelino-Camelia, Gravity-wave interferometers as quantum-gravity detectors, Nature (London) 398, 216 (1999).

[8] G. Amelino-Camelia, Gravity wave interferometers as probes of a low-energy effective quantum gravity, Phys. Rev. D 62, 024015 (2000).
[9] F. Dyson, Is a graviton detectable? Int. J. Mod. Phys. A 28, 1330041 (2013).

[10] E. P. Verlinde and K. M. Zurek, Observational signatures of quantum gravity in interferometers, arXiv:1902.08207.

[11] E. Verlinde and K. M. Zurek, Spacetime fluctuations in AdS/CFT, J. High Energy Phys. 04 (2020) 209.

[12] T. Guerreiro, Quantum effects in gravity waves, Classical Quantum Gravity 37, 155001 (2020).

[13] R. P. Feynman and F. L. Vernon, Jr., The theory of a general quantum system interacting with a linear dissipative system, Ann. Phys. (N.Y.) 24, 118 (1963).

[14] E. Calzetta and B. L. Hu, Noise and fluctuations in semiclassical gravity, Phys. Rev. D 49, 6636 (1994).

[15] B. L. Hu, Stochastic gravity, Int. J. Theor. Phys. 38, 2987 (1999).

[16] K. S. Thorne, Nonradial pulsation of general-relativistic stellar models. IV. The weakfield limit, Astrophys. J. 158, 997 (1969).

[17] W. L. Burke, Gravitational radiation damping of slowly moving systems calculated using matched asymptotic expansions, J. Math. Phys. (N.Y.) 12, 401 (1971).

[18] S. Chandrasekhar and F. Paul Esposito, The 2 $\frac{1}{2}$-postNewtonian equations of hydrodynamics and radiation reaction in general relativity, Astrophys. J. 160, 153 (1970).

[19] Y. Mino, M. Sasaki, and T. Tanaka, Gravitational radiation reaction to a particle motion, Phys. Rev. D 55, 3457 (1997).

[20] R. P. Feynman, R. B. Leighton, and M. Sands, The Feynman Lectures on Physics (Basic Books, 1963), Chap. 28, https:// feynmanlectures.caltech.edu/.

[21] B. P. Abbott et al. (LIGO Scientific and Virgo Collaborations), A guide to LIGO-Virgo detector noise and extraction of transient gravitational-wave signals, Classical Quantum Gravity 37, 055002 (2020).

[22] J. Aasi et al. (LIGO Scientific Collaboration), Advanced LIGO, Classical Quantum Gravity 32, 074001 (2015).

[23] S. Hawking, Black hole explosions, Nature (London) 248, 30 (1974).

[24] L. P. Grishchuk and Y. V. Sidorov, Squeezed quantum states of relic gravitons and primordial density fluctuations, Phys. Rev. D 42, 3413 (1990).

[25] A. Albrecht, P. Ferreira, M. Joyce, and T. Prokopec, Inflation and squeezed quantum states, Phys. Rev. D 50, 4807 (1994).

[26] D. Koks, A. Matacz, and B. L. Hu, Entropy and uncertainty of squeezed quantum open systems, Phys. Rev. D 55, 5917 (1997).

[27] R. J. Glauber, Coherent and incoherent states of the radiation field, Phys. Rev. 131, 2766 (1963). 